\title{
The Number of Prime Divisors of Certain Mersenne Numbers*
}

\author{
By John R. Ehrman
}

It has been conjectured by Gillies [1] that if $M_{p}=2^{p}-1$ is the Mersenne number for some prime $p$, and if $A<B \leqq \sqrt{ } M_{p}$ as $B / A$ and $M_{p} \rightarrow \infty$, then the number of prime divisors of $M_{p}$ in the interval $[A, B]$ is Poisson distributed, with mean

$$
\begin{aligned}
& m \sim \log (\log B / \log A) \quad \text { if } \quad A \geqq 2 p, \text { or } \\
& m \sim \log (\log B / \log 2 p) \text { if } A<2 p .
\end{aligned}
$$

It is the purpose of this paper to describe two tests of a modified form of this conjecture.

It is known that all divisors of $M_{p}$ must be of the form $2 k p+1$ and simultaneously of the form $8 k^{\prime} \pm 1$, where $k$ and $k^{\prime}$ are arbitrary integers. Also, the prime divisors of $M_{p}$ may be of one of the forms $4 n+1$ or $4 n+3$. Thus if $p=$ $4 n+1$, the smallest possible divisor $q$ is $6 p+1$, and if $p=4 n+3$, the smallest possible divisor is $q=2 p+1$. Thus Eq. (1) is modified slightly: the expected number of prime divisors of $M_{p}$ in the interval $[Q, B]$, where $Q$ is not less than the smallest possible divisor of $M_{p}, Q<B \leqq \sqrt{ } M_{p}$, and as $B / Q, M_{p} \rightarrow \infty$, is Poisson distributed with mean

$$
m_{Q} \sim \log (\log B / \log Q) .
$$

Since the observed results in a group are drawn from two populations corresponding to the two forms of $p$, there is a question as to what value $m$ should be used for the estimated mean number of divisors. It would be possible, for example, to separate the two populations and test the samples independently. It was felt, however, that a fuller test of the applicability of the conjecture (2) could be made by testing all primes with no distinction as to form.

In calculating an estimate of the mean $m$ to be used in statistical tests, it was noted that

(a) the sum of two independent random variables from Poisson distributions with parameters $m_{1}$ and $m_{2}$ has a Poisson distribution with parameter $\left(m_{1}+m_{2}\right)$;

(b) if $\pi(x ; k, t)$ is the number of primes $p \equiv t(\bmod k)$ which do not exceed $x$, and if $(k, t)=1$, then [2]

$$
\pi(x ; k, t) \sim \pi(x) / \phi(k) .
$$

This means that one may expect nearly equal numbers of primes of the forms $4 n+1$ and $4 n+3$ in a large sample of primes; this is the justification for not distinguishing the primes as to form.

Thus an unbiased asymptotic estimate of the mean may be taken to be

Received November 9, 1966. Revised February 13, 1967.

* Work supported by U. S. Atomic Energy Commission. 


$$
m=\frac{1}{2}\left(m_{2 p+1}+m_{6 p+1}\right) .
$$

Thus, for example, in the interval $100000<p<102500$, it is found that $m_{2 p+1}=$ 0.5645 and $m_{\mathrm{ep}+1}=0.4784$, so that the Mersenne number corresponding to a prime drawn at random in the interval would be expected to have an average of 0.52 divisors less than $2^{31}$.

The tests performed on the results given in [3] were a test of the mean number of divisors, and a test of their Poisson distribution. Because the change in $p$ over each of the intervals tested is relatively small, the value of $p$ used in computing $m$ from Eq. (3) was simply the midpoint of the interval in $p$ from which the sample was drawn.

A program was written for an IBM System/360 (Model 50) computer which tested for divisors of $M_{p}$ using the congruence

$$
2^{p} \equiv 1 \quad(\bmod q) .
$$

The test was coded [4] in the following manner:

1. In binary form, $p=\sum_{i=0}^{n} a_{i} 2^{i}$, and $2^{p}=\prod_{i=0}^{n}\left(2^{2^{i}}\right)^{a}$.

2. Let $R_{i} \equiv 2^{2^{i}}(\bmod q) \equiv R_{i-1}^{2}(\bmod q)$, and $S_{j}=\prod_{i=0}^{j}\left(R_{i}\right)^{a_{i}}(\bmod q)$.

Thus $S_{j}$ need be computed from $S_{j-1}$ only if $a_{j}=1$.

3. If $S_{n}=1, q \mid M_{p}$.

4. The first five steps of the calculation may be done in one step by taking the five low-order bits of $p$ to compute $R_{4}=2^{p(\bmod 32)}$.

Divisors $q<2^{31}$ were computed for $100000<p<300000$. To compare the observed distribution of primes with that predicted by Eqs. (2) and (3), the values of $p$ were grouped so that $p$ fell into one of the 80 groups defined by

$$
100000+2500 i<p<102500+2500 i,
$$

$i=0(1) 79$. In each group, the total number of primes observed and the number of primes with $j$ divisors were counted. These results are tabulated in Table I. For each $p$ which has one or more divisors $q<2^{31}$, the value of $p$ and the associated values of $k=(q-1) / 2 p$ are tabulated in [3].

To test the estimate of $m, N$ samples of $p$ were observed between limits $L$ and $U$, where $N=\pi(U)-\pi(L)$, and $L<p<U$. The total number of divisors $T$ was counted, and the sample mean $\bar{x}=T / N$ was computed. The sample variance was found from

$$
s^{2}=\frac{1}{N} \sum_{j=1}^{N}{D_{j}}^{2}-(\bar{x})^{2}=\frac{1}{N} \sum_{n=1}^{5} n^{2} K_{n}-(\bar{x})^{2},
$$

where $D_{j}$ is the number of divisors observed for the $j$ th prime in the sample, and $K_{n}$ is the number of Mersenne numbers in the interval with $n$ divisors. (Because of the method used, no tests were made for multiple factors.) As the number of observations becomes large, it is expected that the variable

$$
t=(N-1)^{1 / 2}(\bar{x}-m) / s
$$

should become normally distributed $(0,1)$. The observed values of $N, D$, and $t$ for each group are given in Table I. The expected number of divisors $E$ is simply the product of $m$ and $N$. 


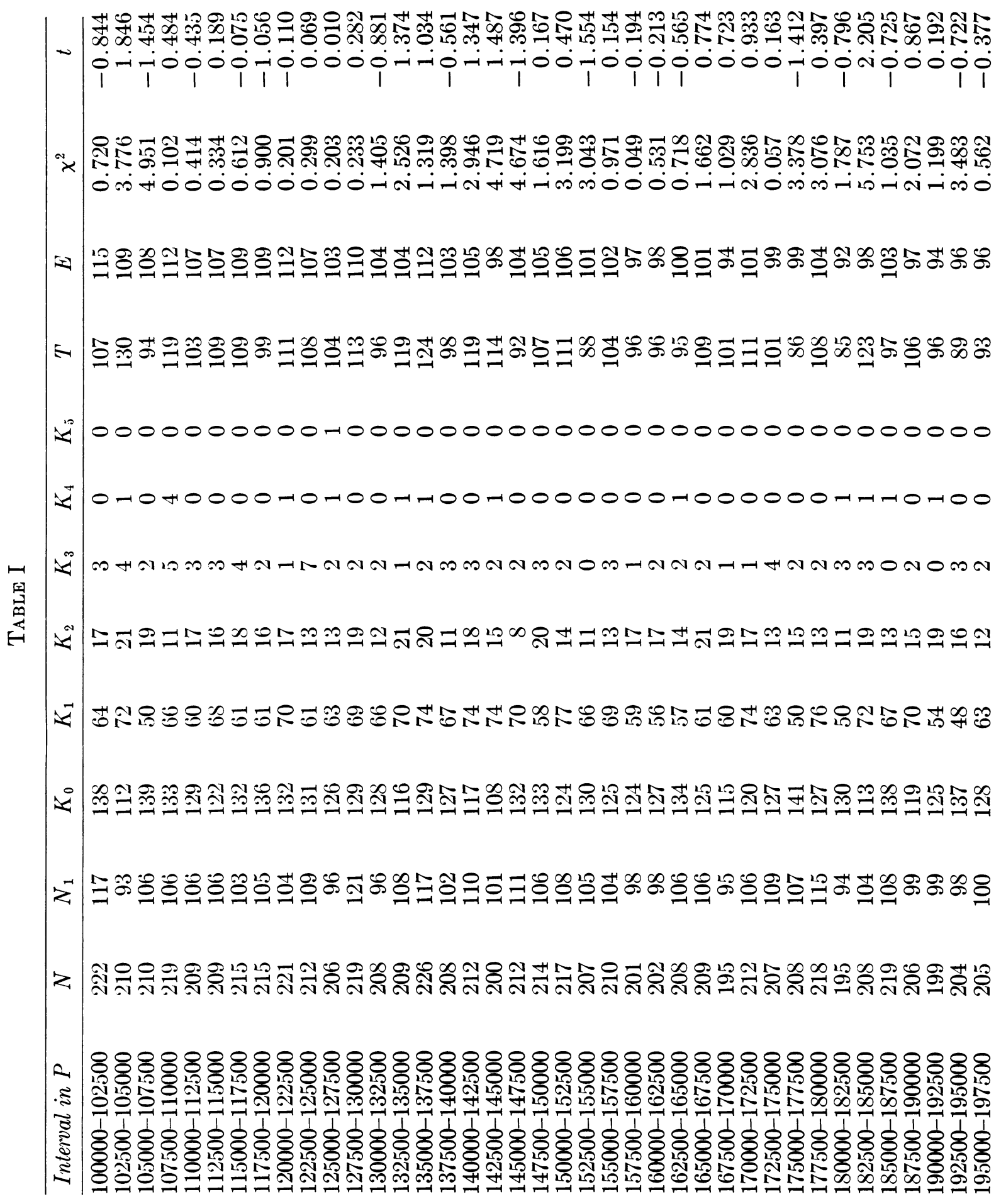




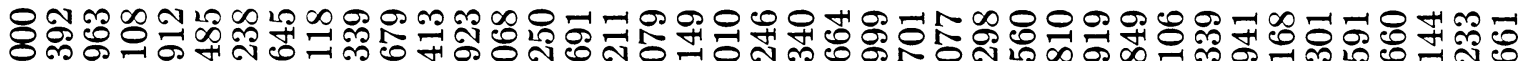

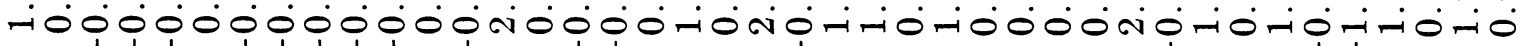

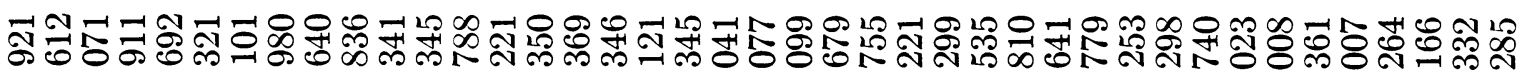

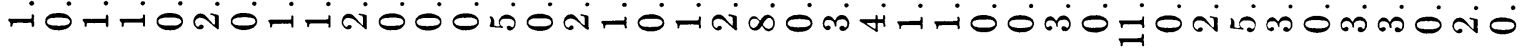

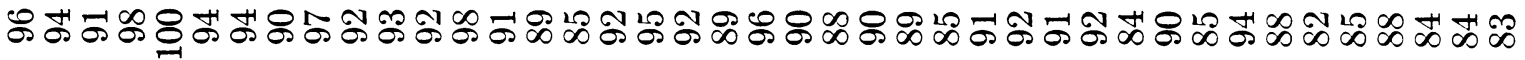

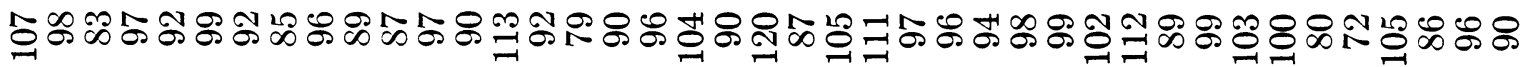

00000000000000000000000000000000000000000

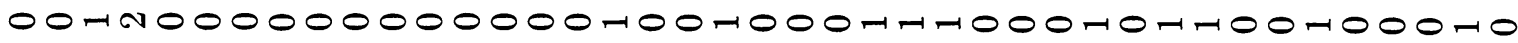

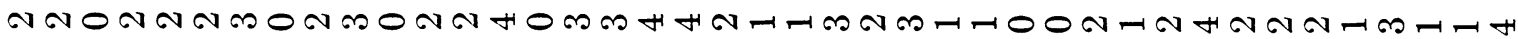

กサー

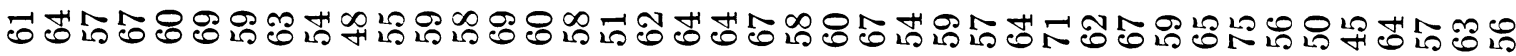

๑ี่ง

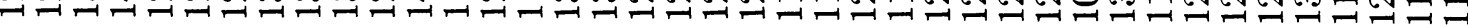

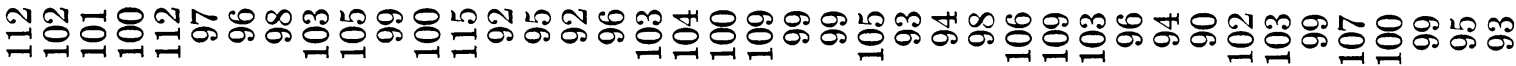

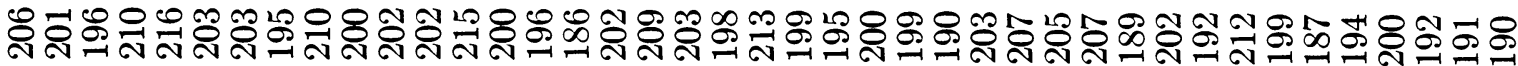

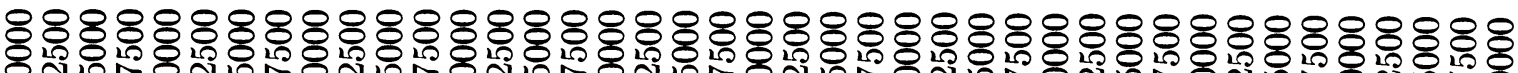

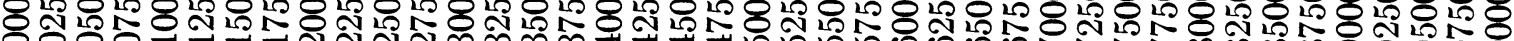
สำล

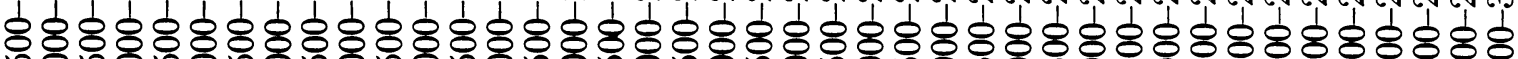

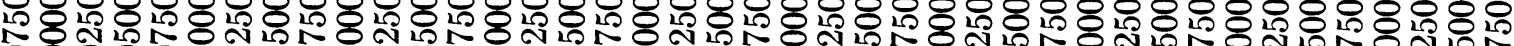
O O O

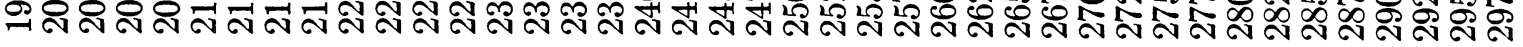


To test the hypothesis of Poisson distribution, a chi-squared test was performed on the observed distribution of divisors. The counts were put in three classes: no divisors, one divisor, and two or more divisors; the numbers of primes with $i$ divisors are listed in the columns $K_{i}$ for $i=0(1) 5$. (See also reference [5].) The computed values of chi-squared for each of the eighty groups are given in Table $\mathrm{I}$. The values of chi-squared were computed from the formula

$$
\begin{aligned}
\chi^{2}= & \left(N e^{-m}-K_{0}\right)^{2}+\left(N m e^{-m}-K_{1}\right)^{2} \\
& +\left(N\left(1-e^{-m}-m e^{-m}\right)-K_{2}-K_{3}-K_{4}-K_{5}\right)^{2},
\end{aligned}
$$

and are given in Table $\mathrm{I}$ in the column headed $\chi^{2}$.

To test for the possibility that distinguishing between primes of the form $4 n+1$ and $4 n+3$ might lead to significantly different results, $t$ and $\chi^{2}$ were also computed for $m=(1 / N)\left[N_{1} m_{6 p+1}+\left(N-N_{1}\right) m_{2 p+1}\right]$, where $N_{1}$ is the number of primes $p \equiv 1(\bmod 4)$ observed in the interval. The average values of $t$ and $\chi^{2}$ obtained were slightly larger than those given at the end of Table I.

A comparison of the expected and observed distributions of $t$ and $\chi^{2}$ is given in Table II. The agreement is seen to be satisfactory.

\section{TABLE II}

Observed distribution of $t$ and chi-squared.

In both cases, the expected number of values in the ranges indicated is 10.

\begin{tabular}{c|c|c|c}
\hline $\begin{array}{c}\text { Upper Limit } \\
\text { on } t\end{array}$ & $\begin{array}{c}\text { Number } \\
\text { of Values }\end{array}$ & $\begin{array}{c}\text { Upper Limit on } \\
\text { Chi-Squared }\end{array}$ & $\begin{array}{c}\text { Number } \\
\text { of Values }\end{array}$ \\
\cline { 1 - 2 }-1.15 & 5 & 0.266 & 10 \\
-.674 & 11 & 0.576 & 12 \\
-.319 & 7 & 0.940 & 9 \\
0.0 & 10 & 1.386 & 10 \\
+.319 & 13 & 1.962 & 8 \\
+.674 & 8 & 2.772 & 8 \\
+1.15 & 12 & 4.158 & 14 \\
$\infty$ & 14 & $\infty$ & 9 \\
\hline
\end{tabular}

I wish to thank J. C. Butcher for several stimulating and helpful discussions, and the referee for several suggestions.

Stanford Linear Accelerator Center

Stanford University

Stanford, California

1. D. B. Gillies, "Three new Mersenne primes and a statistical theory," Math. Comp., v. 18, 1964, p. 94; Also, Digital Computer Laboratory Report No. 138, Univ. of Illinois, Urbana, Ill. MR 28 \#2990.

2. W. J. LeVeque, Topics in Number Theory, Vol. 2, Addison-Wesley, Reading, Mass., 1956, p. 252. MR 18, 283.

3. J. R. Ehrman, "Prime divisors of Mersenne numbers," TN-66-40, Stanford Linear Accelerator Center, Stanford, Calif.

4. Sidney Kravitz, "Divisors of Mersenne numbers 10,000 $<p<15,000$," Math. Comp., v. 15, 1961, p. 292. MR 23 \#A833.

5. Review No. 113, Math. Comp., v. 19, 1965, p. 686. 